\title{
Spatial and spin symmetry breaking in semidefinite-programming-based Hartree-Fock theory
}

\author{
Daniel R. Nascimento ${ }^{1}$ and A. Eugene DePrince III ${ }^{1}$ \\ ${ }^{1}$ Department of Chemistry and Biochemistry, Florida State University, Tallahassee, FL 32306-4390
}

\begin{abstract}
The Hartree-Fock problem was recently recast as a semidefinite optimization over the space of rank-constrained two-body reduced-density matrices (RDMs) [Phys. Rev. A 89, 010502(R) (2014)]. This formulation of the problem transfers the non-convexity of the Hartree-Fock energy functional to the rank constraint on the two-body RDM. We consider an equivalent optimization over the space of positive semidefinite one-electron RDMs (1-RDMs) that retains the non-convexity of the Hartree-Fock energy expression. The optimized 1-RDM satisfies ensemble $N$-representability conditions, and ensemble spin-state conditions may be imposed as well. The spin-state conditions place additional linear and nonlinear constraints on the 1-RDM. We apply this RDM-based approach to several molecular systems and explore its spatial (point group) and spin $\left(\hat{S}^{2}\right.$ and $\left.\hat{S}_{3}\right)$ symmetry breaking properties. When imposing $\hat{S}^{2}$ and $\hat{S}_{3}$ symmetry but relaxing point group symmetry, the procedure often locates spatial-symmetry-broken solutions that are difficult to identify using standard algorithms. For example, the RDM-based approach yields a smooth, spatial-symmetrybroken potential energy curve for the well-known $\mathrm{Be}-\mathrm{H}_{2}$ insertion pathway. We also demonstrate numerically that, upon relaxation of $\hat{S}^{2}$ and $\hat{S}_{3}$ symmetry constraints, the RDM-based approach is equivalent to real-valued generalized Hartree-Fock theory.
\end{abstract}

\section{INTRODUCTION}

Hartree-Fock theory holds an important place in quantum chemistry. It seldom provides a quantitative description of electronic structure, but it serves as a useful starting point for more sophisticated electronic structure methods, such as coupled-cluster theory [1-4]. The canonical form of the Hartree-Fock problem is that of Roothan [5] and Hall [6], which involves the repeated diagonalization of the Fock matrix. When combined with convergence acceleration procedures such as the direct inversion of the iterative subspace (DIIS) [7, 8] and fast two-electron repulsion integral generation (using, for example, graphical processing units 9 [12]), Hartree-Fock theory can be routinely applied to molecular systems containing thousands of atoms [11]. Nevertheless, for large enough systems, the diagonalization of the Fock matrix can eventually become problematic and complicates the development of linearly scaling algorithms.

The direct optimization of the one-electron reduceddensity matrix (1-RDM) is an attractive alternative to the iterative solution of the Roothan-Hall equations for the molecular orbital coefficient matrix; this idea has been widely explored since the 1950s 13 17. The most desirable feature of a density-matrix-based approach is that it avoids the diagonalization of the Fock matrix, thereby facilitating the development of linearly scaling algorithms [18 21]. An immediate drawback, however, is that the 1-RDM associated with the lowest possible energy does not correspond to any antisymmetrized $N$ electron wave function, let alone one comprised of a single Slater determinant. To obtain a physically meaningful result, one must explicitly consider the $N$-representability of the 1-RDM [22]. Typically, $N$-representability (and idempotency) in density-matrix-based Hartree-Fock is acheived through "purification" of an approximately $\mathrm{N}$ - representable 1-RDM [17]. Alternatively, recent work 23 25] has demonstrated the utility of semidefinite programming (SDP) techniques for this problem. The present work explores the SDP-based strategy.

Veeraraghavan and Mazziotti [23] recently recast the Hartree-Fock problem as a constrained optimization over the space of positive semidefinite two-body matrices. The electronic energy was expressed as a linear functional of the 1-RDM and a two-body matrix (denoted ${ }^{2} \mathbf{M}$ in Ref. 23) that is related to the 1-RDM through a contraction. By restricting the rank of ${ }^{2} \mathbf{M}$, one can obtain a rigorous upper bound to the globally optimal Hartree-Fock solution, and this "rank-constrained SDP" 23] optimization has the same formal $\mathcal{O}\left(k^{4}\right)$ cost as the Roothan-Hall form of the problem. Here, $k$ represents the dimension of the one-electron basis set. Alternatively, a lower bound can be obtained by imposing a relaxed set of idempotency constraints on the full-rank matrix. When separate semidefinite optimizations under each set of constraints yield the same 1-RDM, that solution is guaranteed to be the globally optimal one. This guarantee is desirable; the price paid, though, is that the solution of the lower-bound problem requires at least $\mathcal{O}\left(k^{5}\right)$ floating-point operations.

In this work, we consider an alternate representation of the rank-constrained SDP problem that eliminates the consideration of the two-body matrix, ${ }^{2} \mathbf{M}$. The result is a similar SDP algorithm with a non-linear objective function and non-linear constraints on the 1-RDM (in the case where the expectation value of $\hat{S}^{2}$ is constrained). The algorithm retains its formal $\mathcal{O}\left(k^{4}\right)$ scaling, and, like other density-matrix-based Hartree-Fock implementations, it avoids the repeated diagonalization of the Fock matrix. We demonstrate that the SDP-based approach can be applied to several flavors of Hartree-Fock theory, including restricted, unrestricted, and generalized Hartree-Fock (RHF, UHF, and GHF, respectively), depending on which spin symmetries are imposed on the 
1-RDM. We validate the implementation by exploring its spatial (point group) and spin $\left(\hat{S}^{2}\right.$ and $\left.\hat{S}_{3}\right)$ symmetry breaking properties in several molecular systems.

\section{THEORY}

\section{A. Density-matrix-based Hartree-Fock theory}

The electronic energy for the ground state of a manyelectron system is a function of the 1-RDM $(\gamma)$ and the two-electron reduced-density matrix $(2-\mathrm{RDM}, \boldsymbol{\Gamma})$

$$
E=\sum_{p q r s} \Gamma_{q s}^{p r}(p q \mid r s)+\sum_{p q} \gamma_{q}^{p} h_{p q}
$$

Here, $(p q \mid r s)$ represents a two-electron repulsion integral in Mulliken notation, $h_{p q}$ represents the sum of the oneelectron kinetic energy and electron-nuclear potential energy integrals, and the indices $p, q, r$, and $s$ run over all spin orbitals. The $1-\mathrm{RDM}$ and $2-\mathrm{RDM}$ are defined as

$$
\begin{aligned}
\gamma_{q}^{p} & =\left\langle\Psi\left|\hat{a}_{p}^{\dagger} \hat{a}_{q}\right| \Psi\right\rangle, \\
\Gamma_{q s}^{p r} & =\frac{1}{2}\left\langle\Psi\left|\hat{a}_{p}^{\dagger} \hat{a}_{r}^{\dagger} \hat{a}_{s} \hat{a}_{q}\right| \Psi\right\rangle,
\end{aligned}
$$

where $\hat{a}^{\dagger}$ and $\hat{a}$ represent the fermionic creation and annihilation operators of second quantization, respectively. Consider now the cumulant decomposition of the 2-RDM

$$
\Gamma=\gamma \wedge \gamma+\Delta_{2}
$$

where the cumulant matrix, $\boldsymbol{\Delta}_{2}$, represents pure twobody correlations, and the symbol $\wedge$ represents an antisymmetric tensor product (or Grassman product) [26]. By ignoring the cumulant matrix, we arrive at a statistically-independent description of electron motion; the resulting energy expression is equivalent to that from Hartree-Fock theory

$$
E=\frac{1}{2} \sum_{p q r s}\left(\gamma_{q}^{p} \gamma_{s}^{r}-\gamma_{s}^{p} \gamma_{q}^{r}\right)(p q \mid r s)+\sum_{p q} \gamma_{q}^{p} h_{p q}
$$

To obtain the optimal Hartree-Fock 1-RDM, one can invoke the variational principle and minimize the energy given by Eq. (5) with respect to the elements of the 1RDM. Note, however, that this optimization should be carried out under the constraint that the density matrix be idempotent and have a trace equal to the number of electrons. The idempotency condition is a specific manifestation of a more general requirement that any physically meaningful density matrix should correspond to an antisymmetrized $N$-electron wave function. Necessary ensemble $N$-representability conditions require that the eigenvalues of the 1-RDM [the natural orbital (NO) occupation numbers] lie between zero and one and sum to the total number of electrons 22]. The bounds on NO occupation numbers can be enforced by requiring that the 1-RDM be positive semidefinite and related to the one-hole density matrix, $\bar{\gamma}$, by

$$
\gamma_{q}^{p}+\bar{\gamma}_{p}^{q}=\delta_{p q} .
$$

This one-hole density matrix, which must also be positive semidefinite, is defined in second-quantized notation as

$$
\bar{\gamma}_{q}^{p}=\left\langle\Psi\left|\hat{a}_{p} \hat{a}_{q}^{\dagger}\right| \Psi\right\rangle .
$$

The SDP procedure outlined in Sec. ПB enforces these ensemble $N$-representability conditions, rather than the idempotency condition. It is important to note, however, that these conditions do not guarantee idempotency. Fortunately, as discussed in Sec. IV extensive numerical tests indicate that the minimization of the electronic energy given by Eq. (5D) with respect to the elements of the 1-RDM under ensemble $N$-representability constraints always yields an idempotent 1-RDM.

For a non-relativistic Hamiltonian, the exact wave function should have a well-definied total spin $(S)$ and projection of spin $\left(M_{S}\right)$. Hence, one can impose additional conditions on the 1-RDM that fix the particle number and spin state for the system. Particle number and $M_{S}$ can be fixed according to

$$
\operatorname{Tr}\left(\gamma_{\alpha \alpha}\right)=N_{\alpha}
$$

and

$$
\operatorname{Tr}\left(\gamma_{\beta \beta}\right)=N_{\beta},
$$

where the subscripts $\alpha$ and $\beta$ refer to electrons of $\alpha$ and $\beta$ spin, and $\gamma_{\alpha \alpha}$ and $\gamma_{\beta \beta}$ represent the spin-conserving blocks of the 1-RDM, the full structure of which is

$$
\gamma=\left(\begin{array}{ll}
\gamma_{\alpha \alpha} & \gamma_{\alpha \beta} \\
\gamma_{\beta \alpha} & \gamma_{\beta \beta}
\end{array}\right)
$$

Note that the $\gamma_{\alpha \beta}$ and $\gamma_{\beta \alpha}$ blocks are zero if the 1-RDM corresponds to a wave function that is an eigenfunction of $\hat{S}_{3}$. In this case, the total spin quantum number is related to an off-diagonal trace of the $\alpha \beta-\alpha \beta$ spin-block of the 2-RDM [27, 28]; for a statistically-independent pair density, we have

$$
S(S+1)=\frac{1}{2}\left(N_{\alpha}+N_{\beta}\right)+\frac{1}{4}\left(N_{\alpha}-N_{\beta}\right)^{2}-\operatorname{Tr}\left(\gamma_{\alpha \alpha} \gamma_{\beta \beta}\right) .
$$

An ensemble $N$-representable 1-RDM corresponding to a state that is an eigenfunction of $\hat{S}_{3}$ should satisfy Eqs. (6) and (8)-(9); for the 1-RDM to represent an ensemble spin state with total spin, $S$, Eq. (11) should also be satisfied.

Various flavors of Hartree-Fock can be classified according to the symmetries that are preserved by the wave function or density matrix [29 31]. If we relax the spin constraint given by Eq. (11), we arrive at density-matrixbased UHF. If we also lift the constraint that the HartreeFock wave function be an eigenfunction of $\hat{S}_{3}$, we obtain GHF 31 34]. In GHF, the particle number constraints 
of Eqs. (8) and (9) reduce to a single constraint fixing the total particle number

$$
\operatorname{Tr}\left(\boldsymbol{\gamma}_{\alpha \alpha}\right)+\operatorname{Tr}\left(\boldsymbol{\gamma}_{\beta \beta}\right)=N
$$

and $\gamma$ and $\bar{\gamma}$ are no longer guaranteed to have a blockdiagonal spin structure. While $S$ may no longer be a good quantum number, we can still evaluate the expectation value of $\hat{S}^{2}$ according to

$$
\begin{array}{r}
\left\langle\hat{S}^{2}\right\rangle=\frac{3}{4} N+\frac{1}{4}\left[\operatorname{Tr}\left(\gamma_{\alpha \alpha}\right)-\operatorname{Tr}\left(\gamma_{\beta \beta}\right)\right]^{2} \\
-\frac{1}{4} \operatorname{Tr}\left(\gamma_{\alpha \alpha} \gamma_{\alpha \alpha}\right)-\frac{1}{4} \operatorname{Tr}\left(\gamma_{\beta \beta} \gamma_{\beta \beta}\right) \\
+\frac{1}{2} \operatorname{Tr}\left(\gamma_{\alpha \beta}\right) \operatorname{Tr}\left(\gamma_{\beta \alpha}\right)+\operatorname{Tr}\left(\gamma_{\alpha \beta} \gamma_{\beta \alpha}\right)-\operatorname{Tr}\left(\gamma_{\alpha \alpha} \gamma_{\beta \beta}\right) .
\end{array}
$$

\section{B. Semidefinite optimization}

The minimization of the electronic energy given by Eq. (5) subject to the constraints outlined above constitutes a nonlinear semidefinite optimization. We adopt a matrixfactorization-based approach to this problem based upon the "RRSDP" algorithm described in Refs. 35 and 36. The 1-RDM and one-hole density matrix are expressed as contractions of auxiliary matrices, $\boldsymbol{d}$ and $\overline{\boldsymbol{d}}$, as

$$
\gamma_{q}^{p}=\sum_{Q} d_{p}^{Q} d_{q}^{Q}
$$

and

$$
\bar{\gamma}_{q}^{p}=\sum_{Q} \bar{d}_{p}^{Q} \bar{d}_{q}^{Q}
$$

and are thus positive semidefinite by construction. The auxiliary matrices serve as the actual variable quantities in the optimization. Note that the spin structures of $\gamma$ and $\bar{\gamma}$ depend on what spin symmetry is imposed. When $\hat{S}_{3}$ symmetry is enforced, the density matrices consist of two spin-conserving blocks which can be separately factorized as in Eqs. (14) and (15); in this case, $\boldsymbol{d}$ and $\overline{\boldsymbol{d}}$ are also block diagonal, each comprised of two $k \times k$ blocks, where $k$ represents the number of spatial basis functions. When $\hat{S}_{3}$ symmetry is broken, this block structure is lost, and, like the density matrices, the auxiliary matrices are comprised of a single $2 k \times 2 k$ block.

To obtain the optimal $\boldsymbol{d}$ and $\overline{\boldsymbol{d}}$, we minimize the augmented Lagrangian function

$$
L(\boldsymbol{d}, \overline{\boldsymbol{d}})=E(\boldsymbol{d})+\sum_{i}\left[\frac{1}{\mu} C_{i}(\boldsymbol{d}, \overline{\boldsymbol{d}})^{2}-\lambda_{i} C_{i}(\boldsymbol{d}, \overline{\boldsymbol{d}})\right],
$$

with respect to variations in their respective elements. Here, the sum runs over all constraints, $i$, the symbol, $C_{i}(\boldsymbol{d}, \overline{\boldsymbol{d}})$, represents the error in constraint $i$, the symbol, $\lambda_{i}$, represents the corresponding Lagrange multiplier, and $\mu$ is a penalty parameter. The optimization proceeds according to a two-step scheme that is similar to that employed in Refs. 35 and 36:
1. For a set of Lagrange multipliers $\left\{\lambda_{i}^{(n)}\right\}$ and penalty parameter $\mu^{(n)}$, minimize Eq. (16) with respect to the elements of $\boldsymbol{d}$ and $\overline{\boldsymbol{d}}$.

2. Update the Lagrange multipliers

$$
\lambda_{i}^{(n+1)}=\lambda_{i}^{(n)}-2 C_{i}^{(n)}(\boldsymbol{d}, \overline{\boldsymbol{d}}) / \mu^{(n)}
$$

and the penalty parameter

$$
\mu^{(n+1)}=f \mu^{(n)},
$$

where $f$ is defined as

$$
f= \begin{cases}1.0, & \text { if } \frac{\max \left\{\left|C_{i}^{(n)}(\boldsymbol{d})\right|\right\}}{\max \left\{\left|C_{i}^{(n-1)}(\boldsymbol{d})\right|\right\}}<0.25 \\ g, & \text { otherwise. }\end{cases}
$$

The parameter, $g$, is a random number that lies on the interval [0.08:0.12].

Steps 1 and 2 are repeated until the error in the constraints $(\|\boldsymbol{C}(\boldsymbol{d}, \overline{\boldsymbol{d}})\|)$ falls below $10^{-6}$ and the energy changes between iterations by less than $10^{-6} \mathrm{E}_{\mathrm{h}}$. In a typical optimization, there are fewer than 20 of these macroiterations. The parameter, $g$, has been introduced into the RRSDP algorithm because of the non-convex nature of the Hartree-Fock problem. In cases where the algorithm identifies a solution that is not the global solution, $g$ introduces some non-deterministic behavior to facilitate the identifaction of additional solutions in subsequent computations.

In the present implementation, the minimization in Step 1 is achieved using the Limited-memory BroydenFletcher-Goldfarb-Shanno (L-BFGS) method, as implemented in the library liblbfgs. The L-BFGS routine requires the repeated evaluation of the electronic energy, the constraints, and the gradient of the energy and constraints with respect to the elements of $\boldsymbol{d}$ and $\overline{\boldsymbol{d}}$. Evaluating the constraints and the derivative of the constraints with respect to the elements of $\boldsymbol{d}$ and $\overline{\boldsymbol{d}}$ requires only $\mathcal{O}\left(k^{2}\right)$ floating-point operations. The gradient of the energy with respect to the elements of $\boldsymbol{d}$ is given by the matrix product of $\boldsymbol{d}$ and the Fock matrix:

$$
\frac{\partial E}{\partial d_{p}^{Q}}=2 \sum_{q} d_{q}^{Q} F_{q p}
$$

Hence, the rate limiting step in the algorithm is the construction of the Fock matrix, which requires $\mathcal{O}\left(k^{4}\right)$ floating-point operations. Note that, as with the density matrices, the spin structure of the Fock matrix depends on whether or not $\hat{S}_{3}$ symmetry is enforced.

The optimization is performed in the orthonormal basis defined by Löwdin's symmetric orthogonalization, and the initial $\boldsymbol{d}$ and $\overline{\boldsymbol{d}}$ matrices are seeded with random numbers on the interval [-1:1]. At the beginning of an optimization, the L-BFGS step can require hundreds or thousands of Fock matrix builds, but this number significantly 
decreases near convergence of the macroiterations. Obviously, this semidefinite procedure requires that the Fock matrix be built far more times than would be required by a conventional Hartree-Fock algorithm. However, it does have the nice property that the diagonalization of the Fock matrix is avoided completely, unless, of course, the orbital energies themselves are desired at the end of the optimization. Should one wish to devise a linearscaling algorithm based upon the formalism presented herein, it would be desirable to avoid the diagonalization of the overlap matrix as well; in this case, the ensemble $N$-representability conditions for the 1-RDM should be generalized for non-orthogonal orbitals [25].

\section{RESULTS}

\section{A. Broken spatial symmetry}

Artifactual symmetry breaking problems often arise in Hartree-Fock-based descriptions of strongly-correlated systems in which more than one electronic configuration is important in the full configuration interaction (CI) wave function. Small et al. 37 recently demonstrated that complex orbitals resolve this issue for some wellknown RHF-based cases. An illustrative example considered in Ref. 37 is the potential energy curve (PEC) for the $\mathrm{C}_{2 \mathrm{v}}$ insertion of Be into $\mathrm{H}_{2}$, which has also served as a popular model for multireference correlation studies 37 47]. Figure 1(a) illustrates the PEC as a function of the Be- $\mathrm{H}_{2}$ distance, $x$, with the geometry (in units of $\AA$ ) defined as

$$
\begin{aligned}
& \text { Be: }(0.0,0.0,0.0), \\
& \text { H: } \quad(x, 1.344-0.46 x, 0.0), \\
& \text { H: } \quad(x,-1.344+0.46 x, 0.0) .
\end{aligned}
$$

The curve labeled SDP-RHF was generated using the present density-matrix-based algorithm while imposing $\hat{S}^{2}$ [Eq. (11)] and $\hat{S}_{3}$ symmetry [Eqs. (8)-(9)]. Here, the one-electron basis set was cc-pVDZ, and we employed the density fitting approximation to the two-electron repulsion integrals (using the def2-TZVP-JK auxiliary basis set). Full CI computations employed conventional 4index integrals.

As discussed in Ref. 37, the dominant configuration in the full CI ground state is $\left(1 \mathrm{a}_{1}\right)^{2}\left(2 \mathrm{a}_{1}\right)^{2}\left(1 \mathrm{~b}_{2}\right)^{2}$ at $x=0.0$ $\AA$ (the colinear geometry) and $\left(1 \mathrm{a}_{1}\right)^{2}\left(2 \mathrm{a}_{1}\right)^{2}\left(3 \mathrm{a}_{1}\right)^{2}$ at $x=2.1 \AA$ (separated $\mathrm{Be}+\mathrm{H}_{2}$ ). Small et al. demonstrated that complex orbitals yield a PEC that smoothly interpolates between the two RHF limits, avoiding the cusp where these two configurations become degenerate. We see here that the present algorithm, which employs real orbitals and density matrices, also yields a smooth PEC, and inspection of the $1-\mathrm{RDM}$ reveals that this smoothness is achieved through a break in spatial symmetry. Consider the point in the multireference region of the PEC at $x=1.5 \AA$. If we represent the optimized spin-free
1-RDM $\left(\gamma_{\alpha \alpha}+\gamma_{\beta \beta}\right)$ from the present procedure in the basis of symmetry-pure orbitals obtained from conventional RHF with the configuration, $\left(1 \mathrm{a}_{1}\right)^{2}\left(2 \mathrm{a}_{1}\right)^{2}\left(3 \mathrm{a}_{1}\right)^{2}$, the first $4 \times 4$ block has the following structure:

$\begin{array}{lrrrr} & \phi_{1 \mathrm{a}_{1}} & \phi_{2 \mathrm{a}_{1}} & \phi_{3 \mathrm{a}_{1}} & \phi_{1 \mathrm{~b}_{2}} \\ & & & & \\ \phi_{1 \mathrm{a}_{1}} & 2.00 & 0.00 & 0.00 & 0.00 \\ \phi_{2 \mathrm{a}_{1}} & 0.00 & 1.98 & -0.13 & 0.08 \\ \phi_{3 \mathrm{a}_{1}} & 0.00 & -0.13 & 0.68 & 0.92 \\ \phi_{1 \mathrm{~b}_{2}} & 0.00 & 0.08 & 0.92 & 1.27\end{array}$

There is significant mixing between orbitals of $a_{1}$ and $b_{2}$ symmetry, which suggests that the symmetry of the overall wave function has been reduced to at most $\mathrm{C}_{\mathrm{s}}$. Even when fully neglecting spatial symmetry in a conventional RHF computation (using, for example, the implementation in PSI4 48]), one in general locates either one spatially-pure state or another, rather than the spatially contaminated global solution.

Figure1(b) illustrates the same curves generated using second-order perturbation theory (MP2). The MP2 computations built upon orbitals from density-matrix-based Hartree-Fock (which are the eigenfunctions of $\gamma$ ) yield a PEC that interpolates between distinct RHF-based MP2 curves with $\left(1 \mathrm{a}_{1}\right)^{2}\left(2 \mathrm{a}_{1}\right)^{2}\left(1 \mathrm{~b}_{2}\right)^{2}$ and $\left(1 \mathrm{a}_{1}\right)^{2}\left(2 \mathrm{a}_{1}\right)^{2}\left(3 \mathrm{a}_{1}\right)^{2}$ reference functions. Here, however, the curve in the multireference region is not smooth; there is a distinct kink near $x=1.5 \AA$. The broken symmetry MP2 curve also displays a pronounced asymmetry in the multireference region; this behavior is quite similar to that of MP2 built upon complex RHF orbitals, as presented in Ref. 37.

Veeraraghavan and Mazziotti [23] noted similar symmetry-breaking behavior in other challenging multireference problems, such as the dissociation of molecular nitrogen, within their rank-constrained SDP approach to Hartree-Fock theory. This particular symmetry breaking problem has been repeatedly studied using more conventional Hartree-Fock algorithms as well 37, 49, 50]. Figure 2 illustrates conventional RHF and density-matrix-based Hartree-Fock PECs for the dissociation of $\mathrm{N}_{2}$. The RHF curves correspond to configurations that differ only in the occupation of the $\pi\left(1 b_{3 u}\right.$ symmetry) and $\pi^{*}\left(1 b_{3 g}\right.$ symmetry) orbitals. As above, the PEC labeled SDP-RHF was generated using the present approach while enforcing both $\hat{S}^{2}$ and $\hat{S}_{3}$ symmetry, and we observe the same spatial symmetry breaking behavior reported elsewhere. At equilibrium and dissociation, the density-matrix-based approach yields solutions with energies that agree with those of one of the spatially pure RHF configurations or the other. However, for N-N bond lengths in the range of $1.5-2.5 \AA$, we obtain solutions that are lower in energy than both configurations; this stabilization is achieved via a break in the symmetry of the $\pi / \pi^{*}$ orbitals. 
FIG. 1: Potential energy curves for the $\mathrm{C}_{2 \mathrm{v}}$ insertion of Be into $\mathrm{H}_{2}$ in the cc-pVDZ basis set. The curves are shifted such that the energy of the colinear geometry is $0 \mathrm{kcal} \mathrm{mol}^{-1}$. Results are provided at the (a) RHF [using conventional and densitymatrix-based (SDP-RHF) approaches] and (b) MP2 levels of theory. MP2 results were obtained using orbitals from both approaches (indicated in square brackets).

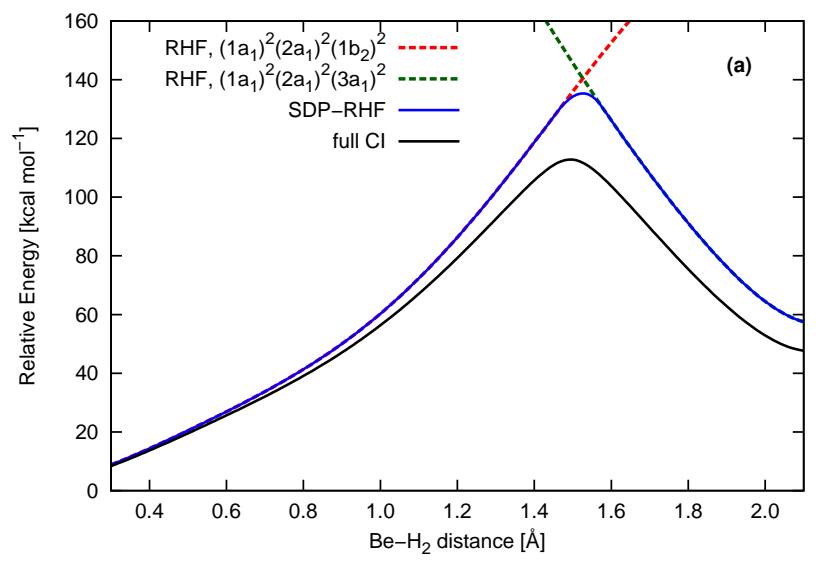

FIG. 2: Potential energy curves for the dissociation of $\mathrm{N}_{2}$ in the cc-pVDZ basis set. ${ }^{a}$

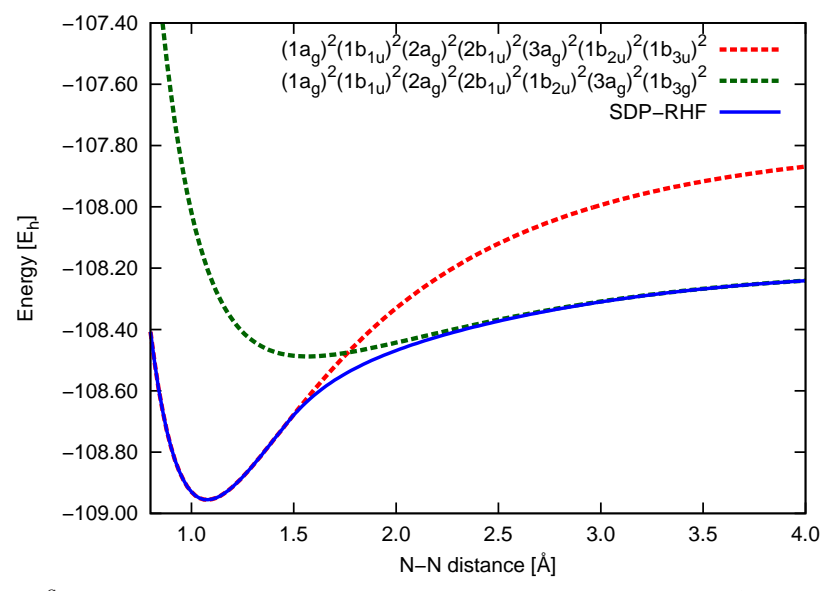

${ }^{a}$ RHF and SDP-RHF computations employ the density fitting approximation and the cc-pVDZ-JK auxiliary basis set.

\section{B. Broken spin symmetry}

While it is desirable for the Hartree-Fock wave function to retain all of the symmetries of the exact wave function, it is often useful, as in the case of describing molecular dissociation, to lift constraints on its spin symmetry $\left(\hat{S}^{2}\right)$. For example, it is well known that RHF in general does not yield the correct dissociation limit for closed shell molecules; UHF usually delivers size consistent results in such cases, at the expense of retaining $S$ as a good quantum number. Interestingly, even UHF does not yield a size-consistent dissociation limit for some closed-shell molecules, such as $\mathrm{CO}_{2}$ [51]. If a truly size-consistent single-determinant method is desired, one must be willing to break additional symmetries in the wave function, such as $\hat{S}_{3}$. In this Section, we explore the spin-symmetry

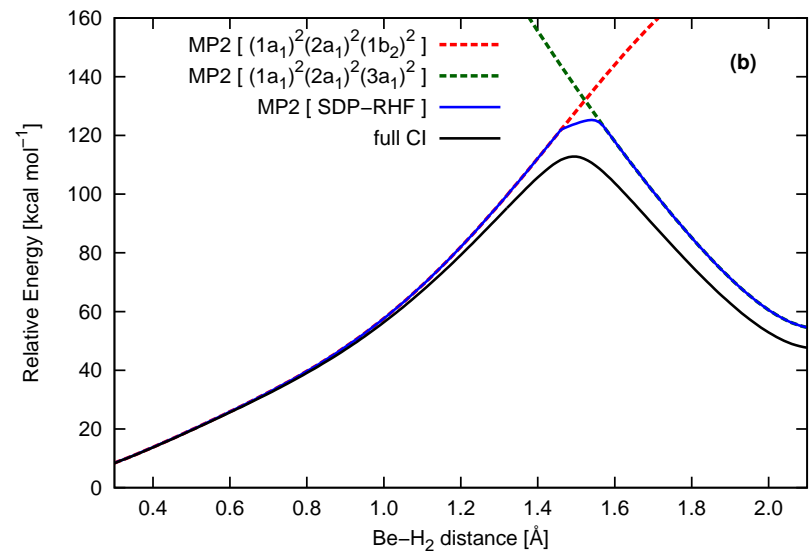

breaking properties of density-matrix-driven UHF and GHF (SDP-UHF and SDP-GHF, respectively) for one well-studied [51] case: the dissociation of molecular oxygen. The 1-RDMs generated from SDP-UHF satisfy ensemble $N$-representability conditions and the $\hat{S}_{3}$ constraints of Eqs. (8)- (9), while those from SDP-GHF satisfy only ensemble $N$-representability conditions. For the remainder of this Section, we use the terms UHF/SDP$\mathrm{UHF}$ and GHF/SDP-GHF interchangeably.

Following Ref. 51, the inability of UHF to yield a sizeconsistent dissociation curve for $\mathrm{O}_{2}$ is easily understood from simple spin arguments. At equilibrium, the ground state of molecular oxygen is the triplet state $\left({ }^{3} \Sigma_{\mathrm{g}}^{-}\right)$, and the ground-state of the dissociation limit involves two oxygen atoms in their triplet states $\left({ }^{3} \mathrm{P}\right)$. The UHF wave function for a given multiplicity is taken to be the highspin determinant, so $\mathrm{O}_{2}$ at equilibrium and each dissociated oxygen atom all have $M_{S}=1$. At dissociation, the two $M_{S}=1$ fragments can only couple to yield states of $M_{S}=2$ (the quintet state) and $M_{S}=0$ (the singlet state). Hence, the UHF triplet state cannot connect the ground-state at equilibrium to those (the singlet or quintet) at dissociation.

Figure 3 provides PECs for the dissociation of molecular oxygen corresponding to the lowest-energy UHF singlet, triplet, and quintet states, as well as that for GHF. All computations were performed within the ccpVDZ basis set using the present density-matrix-based approach to Hartree-Fock. As expected, the lowestenergy UHF curve at equilibrium is that for the triplet state; the singlet UHF curve lies about $30 \mathrm{mE}_{\mathrm{h}}$ higher in energy. At dissociation, the singlet and quintet curves become degenerate and are lowest in energy, while the dissociation limit for the triplet lies about $40 \mathrm{mE}_{\mathrm{h}}$ higher in energy. Hence, as simple spin arguments suggest, the UHF triplet PEC does not connect the lowest energy solutions at equilibrium and dissociation. The present re- 
FIG. 3: SDP-UHF and SDP-GHF potential energy curves for the dissociation of $\mathrm{O}_{2}$ in the cc-pVDZ basis set. ${ }^{a}$

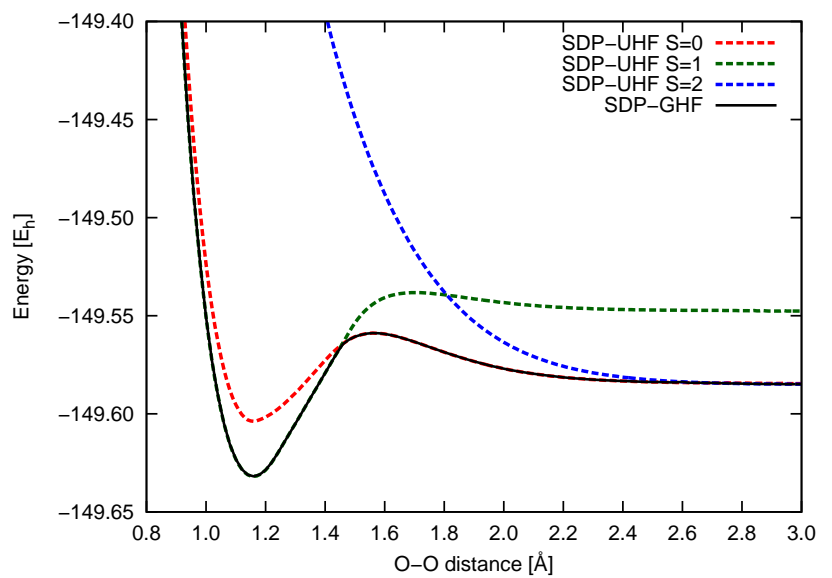

${ }^{a}$ SDP-UHF and SDP-GHF computations employ the density fitting approximation and the cc-pVDZ-JK auxiliary basis set.

sults are in excellent agreement with those of Ref. 51 and provide strong evidence for the numerical equivalence between density-matrix-driven and conventional GHF.

FIG. 4: The region of the potential energy curve for the dissociation of $\mathrm{O}_{2}$ where SDP-GHF provides a lower-energy solution than SDP-UHF (top panel). The value of $\left\langle\hat{S}^{2}\right\rangle$ for SDP-GHF follows those for the SDP-UHF triplet and singlet states at bond lengths less than $1.44 \AA$ and greater than 1.47 $\AA$ A, respectively (bottom panel).
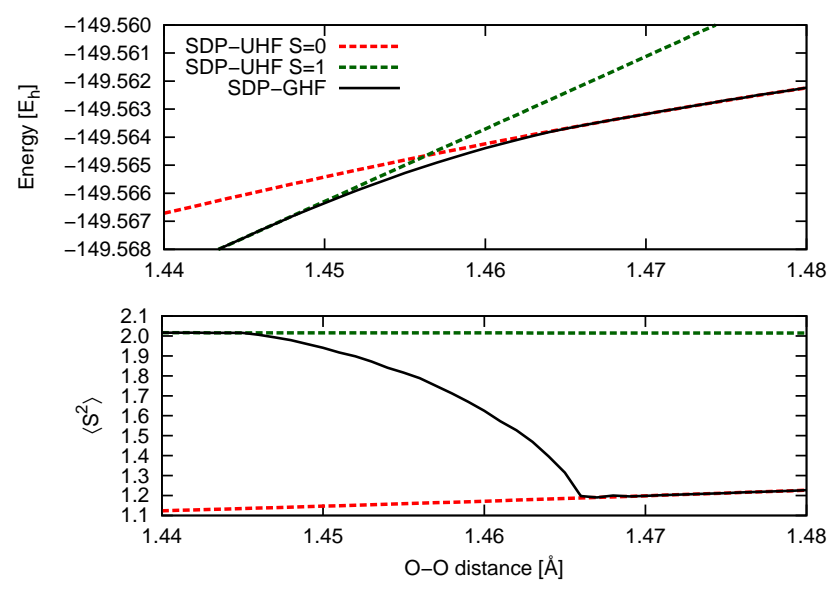

The UHF triplet and singlet states cross at an $\mathrm{O}-\mathrm{O}$ distance slightly less than $1.46 \AA$. The top panel of Fig. 4 provides a more detailed illustration of the relevant PECs in the vicinity of this state crossing. We see that, in the region where the ground state predicted by UHF changes from the triplet state to the singlet state, GHF offers a solution that is lower in energy than either UHF solution. As with the PECs in Fig. 3, this result is in excellent agreement with that from Ref. 51. The lower panel of Fig. 4 illustrates how $\left\langle\hat{S}^{2}\right\rangle$ changes as the GHF PEC interpolates between the UHF triplet and singlet state curves. We note that while the qualitative behavior of $\left\langle\hat{S}^{2}\right\rangle$ in this region resembles that presented in Ref. 51 , there are quantitative differences between our computed values of $\left\langle\hat{S}^{2}\right\rangle$ and theirs. For example, our computations indicate that the expecation value of $\hat{S}^{2}$ merges with that of the UHF singlet curve at an $\mathrm{O}-\mathrm{O}$ distance less than $1.47 \AA$, while the GHF and UHF values of $\left\langle\hat{S}^{2}\right\rangle$ in Ref. 51] merge closer to $1.48 \AA$. Nonetheless, the results are quite similar.

\section{CONCLUSIONS}

We have considered the properties of a nonlinear representation of the rank-constrained semidefinite programming algorithm [23] for the Hartree-Fock problem. The rate limiting step of the SDP-based approach is the formation of the Fock matrix, so it shares the same formal $\mathcal{O}\left(k^{4}\right)$ scaling of the usual Roothan-Hall formulation of the problem. We demonstrated that several flavors of Hartree-Fock theory (e.g. RHF, UHF, GHF) can be implemented within this density-matrix-based formalism depending on which spin symmetry conditions we choose to enforce, and we validated our implementation by studying several spatial and spin symmetry breaking problems.

Interestingly, each flavor of Hartree-Fock theory is recovered without explicitly enforcing any constraints on the idempotency of the 1-RDM. We only enforce ensemble $N$-representability conditions, which apply to both correlated and uncorrelated 1-RDMs alike. This result contrasts with conventional approaches to densitymatrix-based Hartree-Fock theory which rely on purification strategies to obtain idempotent density matrices. We note that we have verified numerically that the optimized 1-RDMs were indeed idempotent in every computation performed in this work. We can rationalize the idempotency of our 1-RDMs by considering the structure of the corresponding statistically independent pair density $(\boldsymbol{\Gamma}=\gamma \wedge \gamma)$. It can be show that the trace of such a 2-RDM is minimized in the case of an idempotent 1-RDM and, accordingly, an energy minimization procedure will favor idempotent 1-RDMs because they minimize electron-electron repulsions.

Alternatively, the idempotency of the 1-RDMs can be understood from the perspective of pure-state $N$ representability in RDM functional theory [52]. In this context, Valone noted 53] that the distinction between pure-state and ensemble $N$-representability is unnecessary in the case of the exact RDM functional. More broadly, pure-state $N$-representability can be achieved under ensemble $N$-representability conditions, provided that the RDM functional is an appropriate one (i.e. that the functional is pure-state $N$-representable). Indeed, our observations are consistent with this proposition. The Hartree-Fock energy functional is the RDM functional that arises for a 1-RDM derived from a single Slater determinant; the Hartree-Fock energy functional is thus pure-state $N$-representable. The pure-state 
$N$-representability of the functional leads to additional desirable properties. For example, the trace constraint for GHF that defines the total particle number [Eq. (12)] technically only enforces the expectation value of $N$. To specify the particle number exactly, the variance in $N,\left\langle\hat{N}^{2}\right\rangle-\langle\hat{N}\rangle^{2}$, should vanish. In the case that (i) the 2-RDM is expressed as an antisymmetrized product of the 1-RDM with itself and (ii) the 1-RDM is idempotent, this variance is exactly zero. As discussed in the Appendix, similar arguments can be made for the exact specification of the $M_{S}$ and $S(S+1)$. Hence, with the choice of the Hartree-Fock energy functional as the RDM functional, the application of ensemble $N$-representability and ensemble spin constraints yields pure-state $N$ - and $S$-representable $1-\mathrm{RDM}$.

\section{Appendix A: On the exact specification of $N, M_{S}$, and $S(S+1)$}

In the semidefinite-programming-based approach to Hartree-Fock theory, the particle number and spin state for the system are specified through constraints on the expectation values of $\hat{N}, \hat{S}_{3}$, and $\hat{S}^{2}$. The exact specification of these quantities technically requires that the corresponding variances (e.g. $\left\langle\hat{N}^{2}\right\rangle-\langle\hat{N}\rangle^{2}$ ) be zero. In the case that the $1-\mathrm{RDM}$ is idempotent and $2-\mathrm{RDM}$ can be constructed as an antisymmetrized product of the 1RDM with itself, we can easily show that the variances for $\hat{N}$ and $\hat{S}_{3}$ exactly vanish. The expectation value of the number operator squared leads to

$$
\left\langle\hat{N}^{2}\right\rangle=\langle\hat{N}\rangle+\langle\hat{N}\rangle^{2}-\operatorname{Tr}(\gamma \gamma)
$$

where we have used the fact that, at the Hartree-Fock level of theory, $\boldsymbol{\Gamma}=\gamma \wedge \gamma$, and we have assumed that trace relations such as Eqs. (8) and (9) or Eq. (12) are satisfied. If the 1-RDM is also idempotent, the first and last terms in Eq. (A1) cancel, and the variance is zero. The same analysis holds for number operators corresponding electrons of $\alpha$ and $\beta$ spin individually $\left(\hat{N}_{\alpha}\right.$ and $\hat{N}_{\beta}$, respectively).

The variance in $M_{S}$ is defined by $\left\langle\hat{S}_{3}^{2}\right\rangle-\left\langle\hat{S}_{3}\right\rangle^{2}$, where $\hat{S}_{3}=\frac{1}{2}\left(\hat{N}_{\alpha}-\hat{N}_{\beta}\right)$, and $M_{S}=\left\langle\hat{S}_{3}\right\rangle$, when Eqs. (8) and (9) are satisfied. If the $2-\mathrm{RDM}$ is expressible in terms of the 1-RDM, we have

$$
\begin{aligned}
\left\langle\hat{S}_{3}^{2}\right\rangle & =\frac{1}{4}\left(\left\langle\hat{N}_{\alpha}\right\rangle-\operatorname{Tr}\left(\gamma_{\alpha} \gamma_{\alpha}\right)+\left\langle\hat{N}_{\beta}\right\rangle-\operatorname{Tr}\left(\gamma_{\beta} \gamma_{\beta}\right)\right. \\
& \left.+\left\langle\hat{N}_{\alpha}\right\rangle^{2}+\left\langle\hat{N}_{\beta}\right\rangle^{2}-2\left\langle\hat{N}_{\alpha}\right\rangle\left\langle\hat{N}_{\beta}\right\rangle\right)
\end{aligned}
$$

In the case that $1-\mathrm{RDM}$ is idempotent, Eq. A2 reduces to

$$
\left\langle\hat{S}_{3}^{2}\right\rangle=\frac{1}{4}\left(\left\langle\hat{N}_{\alpha}\right\rangle-\left\langle\hat{N}_{\beta}\right\rangle\right)^{2}=\left\langle\hat{S}_{3}\right\rangle^{2}
$$

FIG. 5: The (a) total energy $\left(E_{h}\right)$, (b) expectation value of $\hat{S}^{2}$, and (c) the Euclidean norm of the error in the pure spin conditions of Eq. A6 for the dissociation of molecular nitrogen in the cc-pVDZ basis set. ${ }^{a}$
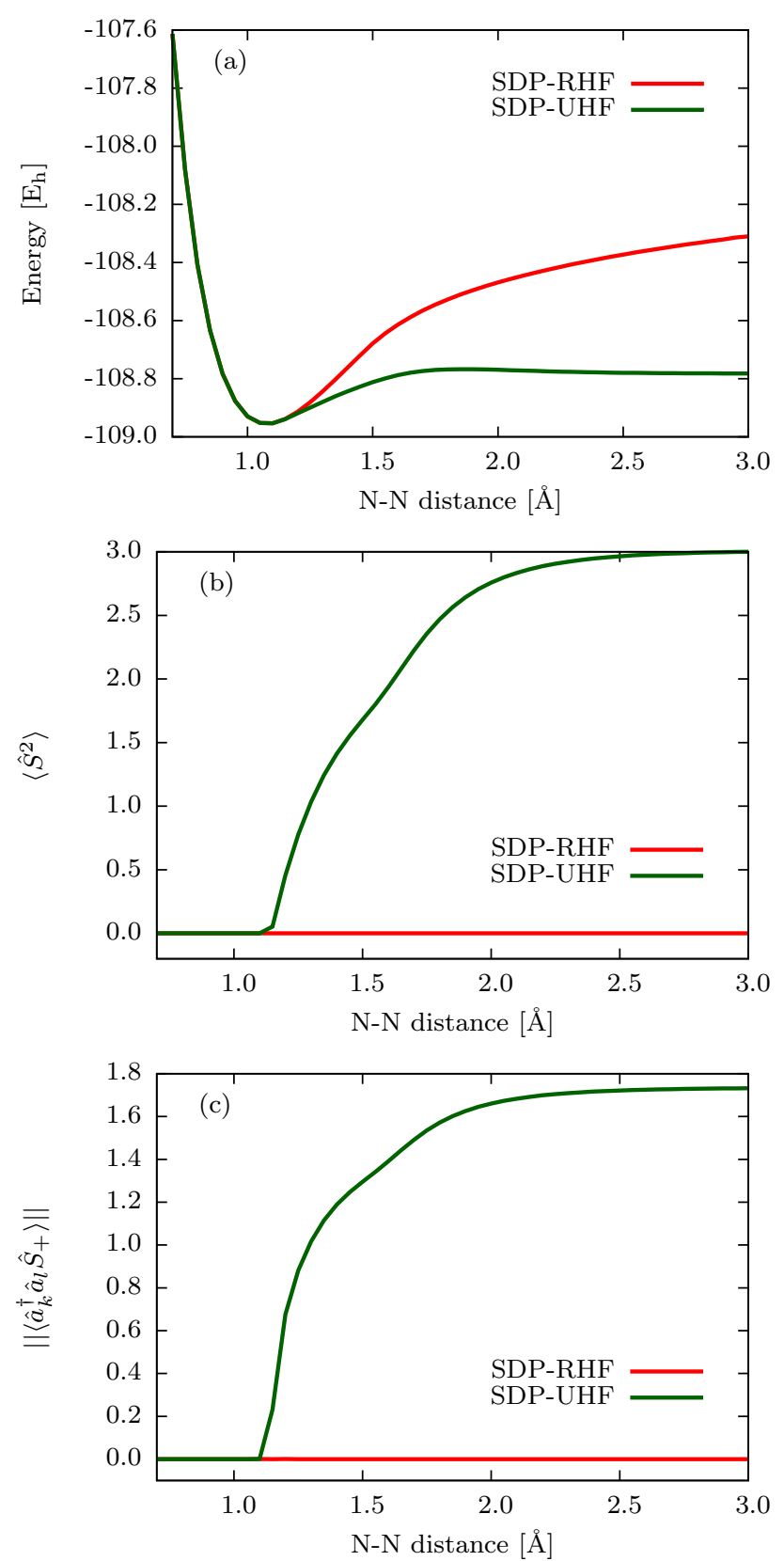

${ }^{a}$ SDP-RHF and SDP-UHF computations employ the density fitting approximation and the cc-pVDZ-JK auxiliary basis set.

and the variance in $M_{S}$ vanishes.

A similar analysis of the variance in $S(S+1)$ is more involved, as the expectation value of $\hat{S}^{4}$ depends upon the four-particle RDM. Instead, we consider the two-particle pure-spin state conditions detailed in Ref. 54, the first of which is a "contraction condition" requiring that any pure spin state, $\left|\Psi^{S M_{S}}\right\rangle$, should satisfy

$$
\left\langle\Psi^{S M_{S}}\left|\hat{a}_{k}^{\dagger} \hat{a}_{l}\left(N \hat{S}_{3}-M_{S} \hat{N}\right)\right| \Psi^{S M_{S}}\right\rangle=0
$$


FIG. 6: The (a) total energy $\left(\mathrm{E}_{\mathrm{h}}\right)$, (b) expectation value of $\hat{S}^{2}$, and (c) the Euclidean norm of the error in the pure spin conditions of Eq. A6 for the dissociation of hydroxyl radical in the cc-pVDZ basis set. ${ }^{a}$
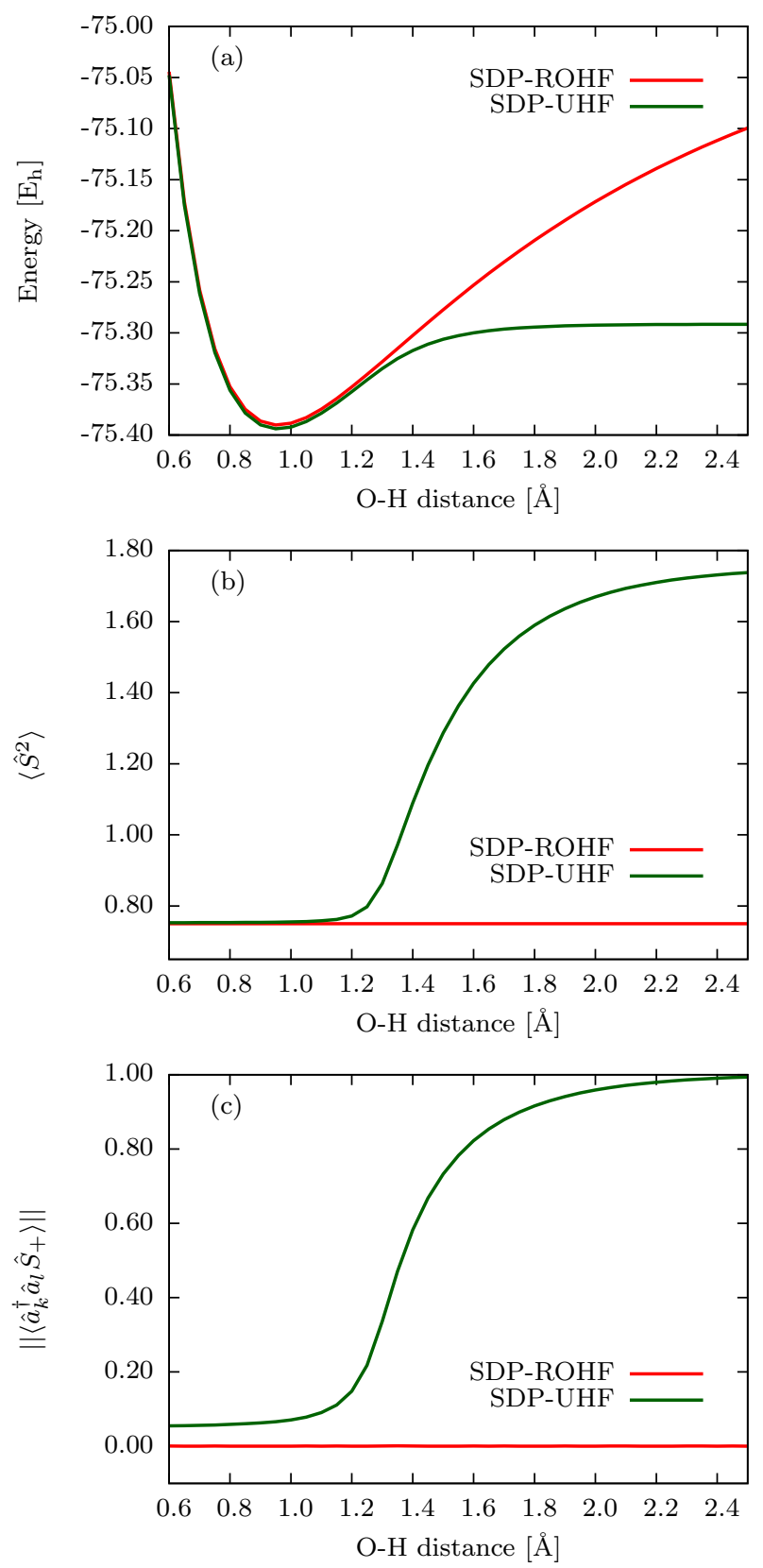

${ }^{a}$ SDP-ROHF and SDP-UHF computations employ the density fitting approximation and the cc-pVDZ-JK auxiliary basis set.

for all spin orbitals, $k$ and $l$. As with the variances above, this condition is automatically satisfied in the case that the $2-\mathrm{RDM}$ is represented as an antisymmetrized product of an idempotent 1-RDM with itself and that Eqs. (8) and (9) are satisfied. A less trival constraint requires that the maximal spin projection for a spin pure state satisfies

$$
\left\langle\Psi^{S M_{S}}\left|\hat{a}_{k}^{\dagger} \hat{a}_{l} \hat{S}_{+}\right| \Psi^{S M_{S}}\right\rangle=0,
$$

for all spin orbitals, $k$ and $l$. For a $1-R D M$ with $\hat{S}_{3}$ symmetry and a 2-RDM expressible in terms of the 1-RDM, this set of constraints reduces to

$$
\forall k, l: \gamma_{l_{\beta}}^{k_{\beta}}-\sum_{p} \gamma_{l_{\alpha}}^{p_{\alpha}} \gamma_{p_{\beta}}^{k_{\beta}}=0,
$$

where the Greek subscripts denote the spin component of the orbital label. These constraints may be satisfied for a singlet state, where $\gamma_{\alpha \alpha}=\gamma_{\beta \beta}$, and the 1-RDM is idempotent. However, these constraints are not obviously satisfied for other spin states. Here, we demonstrate numerically that these constraints are satisfied in SDP-based Hartree-Fock through constraints on the expectation values of $\hat{S}_{3}$ and $\hat{S}^{2}$.

Figure 5(a) illustrates the potential energy curve for the dissociation of molecular nitrogen at the SDP-RHF and SDP-UHF levels of theory. The expectation values of $\hat{S}^{2}$ from RHF and UHF [Fig. [5(b)] diverge as the respective energies diverge, beyond the Coulson-Fischer point. Figure 5(c) shows the norm of the constraints given by Eq. (A6); we can see that these constraints are satisfied by SDP-RHF (the norm is zero), whereas the norm computed from the SDP-UHF 1-RDM becomes quite large beyond the Coulson-Fischer point.

Lastly, we consider a similar analysis for a non-singlet case: the dissociation of the hydroxyl radical. Figure 6 provides results obtained using SDP-based restricted open-shell Hartree-Fock (SDP-ROHF) and SDP-UHF. The 1-RDM obtained from SDP-ROHF satisfies Eqs. (8) and (9) as well as the $\hat{S}^{2}$ constraint of Eq. (11) with $S=0.5$. Panels (b) and (c) of Fig. 6 demonstrate that SDP-ROHF yields the correct value of $S(S+1)$ at all $\mathrm{O}-\mathrm{H}$ distances, and the norm of the errors defined by Eq. A6 are zero for the entire curve as well. The corresponding data for SDP-UHF show clear deviations from the respective values for a pure spin state, as expected.

\section{Acknowledgments:}

This work was supported as part of the Center for Actinide Science and Technology (CAST), an Energy Frontier Research Center funded by the U.S. Department of Energy, Office of Science, Basic Energy Sciences under Award No. de-sc0016568.
[1] J. Č́žžek, J. Chem. Phys. 45, 4256 (1966).

[2] G. D. Purvis and R. J. Bartlett, J. Chem. Phys. 76, 1910 (1982).
[3] K. Raghavachari, G. W. Trucks, J. A. Pople, and M. Head-Gordon, Chem. Phys. Lett. 157, 479 (1989).

[4] R. J. Bartlett and M. Musiał, Rev. Mod. Phys. 79, 291 
(2007).

[5] C. C. J. Roothaan, Rev. Mod. Phys. 23, 69 (1951).

[6] G. G. Hall, Proc. Royal Soc. A 205, 541 (1951).

[7] P. Pulay, Chem. Phys. Lett. 73, 393 (1980).

[8] P. Pulay, J. Comput. Chem. 3, 556 (1982).

[9] K. Yasuda, J. Comput. Chem. 29, 334 (2008).

[10] I. S. Ufimtsev and T. J. Martnez, J. Chem. Theory Comput. 4, 222 (2008).

[11] N. Luehr, I. S. Ufimtsev, and T. J. Martnez, J. Chem. Theory Comput. 7, 949 (2011).

[12] J. Kalinowski, F. Wennmohs, and F. Neese, J. Chem. Theory Comput. 13, 3160 (2017).

[13] P.-O. Löwdin, Phys. Rev. 97, 1490 (1955).

[14] R. McWeeny, Proc. Royal Soc. A 235, 496 (1956).

[15] R. McWeeny, Phys. Rev. 114, 1528 (1959).

[16] R. McWeeny, Phys. Rev. 126, 1028 (1962).

[17] R. McWeeny, Rev. Mod. Phys. 32, 335 (1960).

[18] F. Mauri, G. Galli, and R. Car, Phys. Rev. B 47, 9973 (1993).

[19] X.-P. Li, R. W. Nunes, and D. Vanderbilt, Phys. Rev. B 47, 10891 (1993).

[20] E. B. Stechel, A. R. Williams, and P. J. Feibelman, Phys. Rev. B 49, 10088 (1994).

[21] J. Kussmann, M. Beer, and C. Ochsenfeld, WIREs Comput. Mol. Sci. 3, 614 (2013), ISSN 1759-0884.

[22] A. J. Coleman, Rev. Mod. Phys. 35, 668 (1963).

[23] S. Veeraraghavan and D. A. Mazziotti, Phys. Rev. A 89, 010502 (2014).

[24] S. Veeraraghavan and D. A. Mazziotti, J. Chem. Phys. 140, 124106 (2014).

[25] S. Veeraraghavan and D. A. Mazziotti, Phys. Rev. A 92, $022512(2015)$.

[26] A. J. Coleman and I. Absar, Int. J. Quantum Chem. 18, 1279 (1980).

[27] E. Pérez-Romero, L. M. Tel, and C. Valdemoro, Int. J. Quantum Chem. 61, 55 (1997).

[28] G. Gidofalvi and D. A. Mazziotti, Phys. Rev. A 72, 052505 (2005).

[29] H. Fukutome, Int. J. Quantum Chem. 20, 955 (1981).

[30] J. Stuber and J. Paldus, Fundamental World of Quantum Chemistry, A Tribute Volume to the Memory of Per-Olov Löwdin 1, 67 (2003).

[31] C. A. Jiménez-Hoyos, T. M. Henderson, and G. E. Scuseria, J. Chem. Theory Comput. 7, 2667 (2011).

[32] J. G. Valatin, Phys. Rev. 122, 1012 (1961).
[33] A. K. Kerman and A. Klein, Phys. Rev. 132, 1326 (1963).

[34] M. M. Mestechkin and G. E. Whyman, Int. J. Quantum Chem. 8, 45 (1974).

[35] D. A. Mazziotti, Phys. Rev. Lett. 93, 213001 (2004).

[36] D. A. Mazziotti, ESAIM, Math. Model. Numer. Anal. 41, 249 (2007).

[37] D. W. Small, E. J. Sundstrom, and M. Head-Gordon, J. Chem. Phys. 142, 024104 (2015).

[38] G. D. Purvis, R. Shepard, F. B. Brown, and R. J. Bartlett, Int. J. Quantum Chem. 23, 835 (1983).

[39] U. S. Mahapatra, B. Datta, B. Bandyopadhyay, and D. Mukherjee, Adv. Quantum Chem. 30, 163 (1998).

[40] U. S. Mahapatra, B. Datta, and D. Mukherjee, J. Chem. Phys. 110, 6171 (1999).

[41] F. A. Evangelista, M. Hanauer, A. Köhn, and J. Gauss, J. Chem. Phys. 136, 204108 (2012).

[42] D. I. Lyakh, M. Musiał, V. F. Lotrich, and R. J. Bartlett, Chem. Rev 112, 182 (2012).

[43] J. Brabec, H. J. van Dam, J. Pittner, and K. Kowalski, J. Chem. Phys. 136, 124102 (2012).

[44] Z. Chen and M. R. Hoffmann, J. Chem. Phys. 137, 014108 (2012).

[45] J. P. Coe, D. J. Taylor, and M. J. Paterson, J. Chem. Phys. 137, 194111 (2012).

[46] O. Demel, S. Kedžuch, M. Švaňa, S. Ten-No, J. Pittner, and J. Noga, Phys. Chem. Chem. Phys. 14, 4753 (2012).

[47] C. A. Jiménez-Hoyos, R. Rodríguez-Guzmán, and G. E. Scuseria, J. Chem. Phys. 139, 224110 (2013).

[48] R. M. Parrish, L. A. Burns, D. G. A. Smith, A. C. Simmonett, A. E. DePrince, E. G. Hohenstein, U. Bozkaya, A. Y. Sokolov, R. Di Remigio, R. M. Richard, et al., J. Chem. Theory Comput. 13, 3185 (2017).

[49] X. Li and J. Paldus, J. Chem. Phys. 130, 084110 (2009).

[50] A. J. W. Thom and M. Head-Gordon, Phys. Rev. Lett. 101, 193001 (2008).

[51] C. A. Jiménez-Hoyos, T. M. Henderson, T. Tsuchimochi, and G. E. Scuseria, J. Chem. Phys. 136, 164109 (2012).

[52] M. Piris, in Many-body approaches at different scales: A tribute to Norman H. March on the occasion of his 90th birthday, edited by G. G. N. Angilella and C. Amovilli (Springer, 2018), chap. 22, pp. 283-300.

[53] S. M. Valone, J. Chem. Phys. 73, 1344 (1980).

[54] H. van Aggelen, B. Verstichel, P. Bultinck, D. V. Neck, and P. W. Ayers., J. Chem. Phys. 136, 014110 (2012). 